\title{
Serum PR3-ANCA Is a Predictor of Primary Nonresponse to Anti-TNF-a Agents in Patients with Ulcerative Colitis
}

\author{
Atsushi Yoshida ${ }^{a}$ Katsuyoshi Matsuoka ${ }^{b}$ Fumiaki Ueno $^{a}$ Toshio Morizane $^{a}$ \\ Yutaka Endo ${ }^{\text {a }}$ Toshifumi Hibic \\ ${ }^{a}$ Center for Gastroenterology and Inflammatory Bowel Disease, Ofuna Chuo Hospital, Kamakura, Japan; \\ ${ }^{\mathrm{b}}$ Department of Gastroenterology and Hepatology, Toho University Sakura Medical Center, Chiba, Japan; 'Center \\ for Advanced IBD Research and Treatment, Kitasato Institute Hospital, Kitasato University, Tokyo, Japan
}

\section{Keywords \\ Inflammatory bowel disease · Primary failure · Infliximab · Adalimumab · Golimumab}

\begin{abstract}
Background: Anti-tumor necrosis factor-a (TNF-a) agents are effective for moderately to severely active ulcerative colitis (UC). Nonetheless, a proportion of patients fail to respond to these agents as therapy for induction of remission. Recent studies indicated that perinuclear anti-neutrophil cytoplasmic antibody ( $p$-ANCA) may predict response to anti-TNF- $a$ agents in UC patients. However, whether PR3-ANCA can predict primary nonresponse (PNR) to anti-TNF-a agents has not yet been evaluated. The aim of this study was to examine whether PR3-ANCA can predict PNR to anti-TNF-a in UC patients. Methods: This was a single-center retrospective study. Data were extracted from 50 patients with UC who had measurements of PR3-ANCA and received anti-TNF- $a$ agents for the first time as induction therapy. The primary endpoint of this study was a proportion of patients with PNR stratified by PR3-ANCA positivity. PNR to anti-TNF-a agents was defined as failure to achieve reduction in partial Mayo score by 2 or more points and change to other therapeutics within 6 weeks. Results: Fourteen (28\%) of the 50 patients
\end{abstract}

were PR3-ANCA positive. Seventeen (34\%) of the 50 patients demonstrated PNR. Eleven (78.6\%) of the 14 PR3-ANCA-positive patients demonstrated PNR, while 6 (16.7\%) of the 36 PR3-ANCA-negative patients demonstrated PNR. Multivariate analysis demonstrated that PR3-ANCA positivity was associated with PNR to anti-TNF-a agents (odds ratio 19.29, 95\% Cl: 3.30-172.67; $p=0.002)$. Conclusion: PR3-ANCA positivity can predict PNR to anti-TNF-a agents in UC patients.

(c) 2021 The Author(s)

Published by S. Karger AG, Basel

\section{Introduction}

Ulcerative colitis (UC) is a chronic inflammatory disease of the colon and rectum with repeated cycles of remission and relapse [1]. UC and Crohn's disease (CD) are the 2 major forms of inflammatory bowel disease (IBD). The etiology of UC remains unknown. Treatment is determined according to disease extent and activity. Firstline therapy for patients with mild-to-moderate activity is 5-aminosalicylic acid (5-ASA). Corticosteroids are used for patients who are refractory to 5-ASA or who have moderate-to-severe activity. However, roughly half of patients are resistant to or dependent on corticosteroids. Anti-tumor necrosis factor-alpha (TNF- $\alpha)$ agents are indi-
(C) 2021 The Author(s)

Published by S. Karger AG, Basel

This is an Open Access article licensed under the Creative Commons Attribution-NonCommercial-4.0 International License (CC BY-NC) (http://www.karger.com/Services/OpenAccessLicense), applicable to the online version of the article only. Usage and distribution for commercial purposes requires written permission.
Correspondence to:

Atsushi Yoshida, atushi.y@kza.biglobe.ne.jp 
cated for treatment of patients with moderate-to-severe UC that is refractory to corticosteroids [2-5]. Despite the efficacy of anti-TNF- $\alpha$ agents, $30-40 \%$ of patients do not respond to initial induction dosing of anti-TNF- $\alpha$ agents, known as primary nonresponse (PNR). Several predictors of PNR, including disease activity and serum albumin levels, have been reported. However, none have sufficient predictive capacity for clinical use [6].

Anti-neutrophil cytoplasmic antibodies (ANCAs) are autoantibodies to antigens localized in the cytoplasm of neutrophils [7]. ANCAs are classified into 2 types according to staining patterns on indirect immunofluorescence (IIF): cytoplasmic ANCA (c-ANCA), whereby neutrophil cytoplasm is stained granularly, and perinuclear ANCA (p-ANCA), whereby antigens around the nucleus are stained $[8,9]$. The target antigens of p-ANCA and cANCA were subsequently identified: myeloperoxidase for $\mathrm{p}$-ANCA and protease-3 (PR-3) for c-ANCA. These antigens can be measured using enzyme-linked immunosorbent assay (ELISA) or chemiluminescent enzyme immunoassay (CLEIA), which are more specific and sensitive than IIF.

ANCAs are diagnostic markers for ANCA-associated vasculitis (AAV). In addition to AAV, ANCA positivity is observable in patients with various immunological disorders such as rheumatoid arthritis and primary biliary cholangitis [10]. P-ANCA positivity was also reported in approximately $10-80 \%$ of UC patients not complicated with AAV and can be used as a diagnostic marker for UC [10-13]. A meta-analysis demonstrated the sensitivity and specificity of p-ANCA positivity were 55.3 and $88.5 \%$, respectively, in the differential diagnosis of $\mathrm{UC}$ and $\mathrm{CD}$ [12]. Interestingly, several recent studies reported that $\mathrm{p}$ ANCA can also serve as a predictor of PNR to anti-TNF- $\alpha$ agents in UC patients [14-19].

PR3-ANCA positivity, corresponding to c-ANCA, is also observable in UC patients and was reported to have a sensitivity of $58 \%$ and a specificity of $93 \%$ in the differential diagnosis of UC and CD [20]. However, whether PR3-ANCA can predict PNR to anti-TNF- $\alpha$ agents in UC patients has not yet been examined. The aim of this study was to investigate whether PR3-ANCA is a predictor of PNR to anti-TNF- $\alpha$ agents in UC patients.

\section{Materials and Methods}

\section{Study Design}

This was a retrospective cohort study conducted at a regional IBD center in Japan.
Patients

We included UC patients who received anti-TNF- $\alpha$ agents (infliximab [IFX], adalimumab [ADA], and golimumab [GLM]) for the first time between January 2012 and December 2019 and had available data on serum PR3-ANCA. Diagnosis of UC was made in accordance with Japanese practice guidelines [21].

\section{Treatment}

We administered anti-TNF- $\alpha$ agents at standard dose regimens: IFX was administered at $5 \mathrm{mg} / \mathrm{kg}$ at 0,2 , and 6 weeks and every 8 weeks thereafter; ADA was administered at $160 \mathrm{mg}$ at week $0,80 \mathrm{mg}$ at week 2 , and then $40 \mathrm{mg}$ biweekly; and GLM was administered at $200 \mathrm{mg}$ at week $0,100 \mathrm{mg}$ at week 2, and then at 100 mg every 4 weeks.

\section{Data Collection}

We extracted the following data from medical charts: age, sex, body mass index (BMI), disease extent, disease duration, Mayo score [22], serum albumin levels, hemoglobin ( $\mathrm{Hb}$ ) levels, serum C-reactive protein (CRP) levels, serum PR3-ANCA levels, smoking history, and concomitant medications. PR3-ANCA was measured using CLEIA (Hoken Kagaku, Inc., Yokohama, Japan). PR3ANCA positivity was defined as $3.5 \mathrm{U} / \mathrm{mL}$ or higher.

\section{Outcome Measurements}

The primary outcome of this study was proportion of patients with PNR to anti-TNF- $\alpha$ agents stratified by PR3-ANCA positivity. PNR was defined as failure to achieve reduction in partial Mayo score by 2 or more points and change to other therapies within 6 weeks. Patients whose treatment was changed because of intolerance to anti-TNF- $\alpha$ agents were excluded from analysis. As predictive factors for PNR, we examined age, sex, disease duration, disease extent, BMI, Mayo score, endoscopic Mayo subscore, albumin, $\mathrm{Hb}, \mathrm{CRP}$, smoking habit, and concomitant medications.

\section{Statistical Analysis}

Normally distributed continuous variables are presented as mean $\pm S D$, and nonparametric variables are presented as median (interquartile range $[\mathrm{IQR}]$ ). Normality was assessed with the Shapiro-Wilk test. For statistical analyses, each explanatory variable was compared between those with and without PNR using Student's $t$ test, the $\chi^{2}$ test, or the Wilcoxon rank-sum test. Factors contributing to PNR to anti-TNF- $\alpha$ agents were also analyzed using logistic regression analysis with explanatory variables. PNR prediction performance (sensitivity and specificity) was evaluated for significant factors. For these analyses, we used R functions. $p$ values $<0.05$ were considered statistically significant.

\section{Results}

\section{Patient Demographics}

During the study period, 88 patients received antiTNF- $\alpha$ agents for the first time (IFX: $n=51$ [58\%]; ADA: $n=28$ [32\%]; and GLM: $n=9$ [10\%]). Data on PR3ANCA were available in 51 (58\%) patients. For most patients, PR3-ANCA was measured for differential diagno- 
Table 1. Characteristics of patients enrolled in this study $(N=50)$

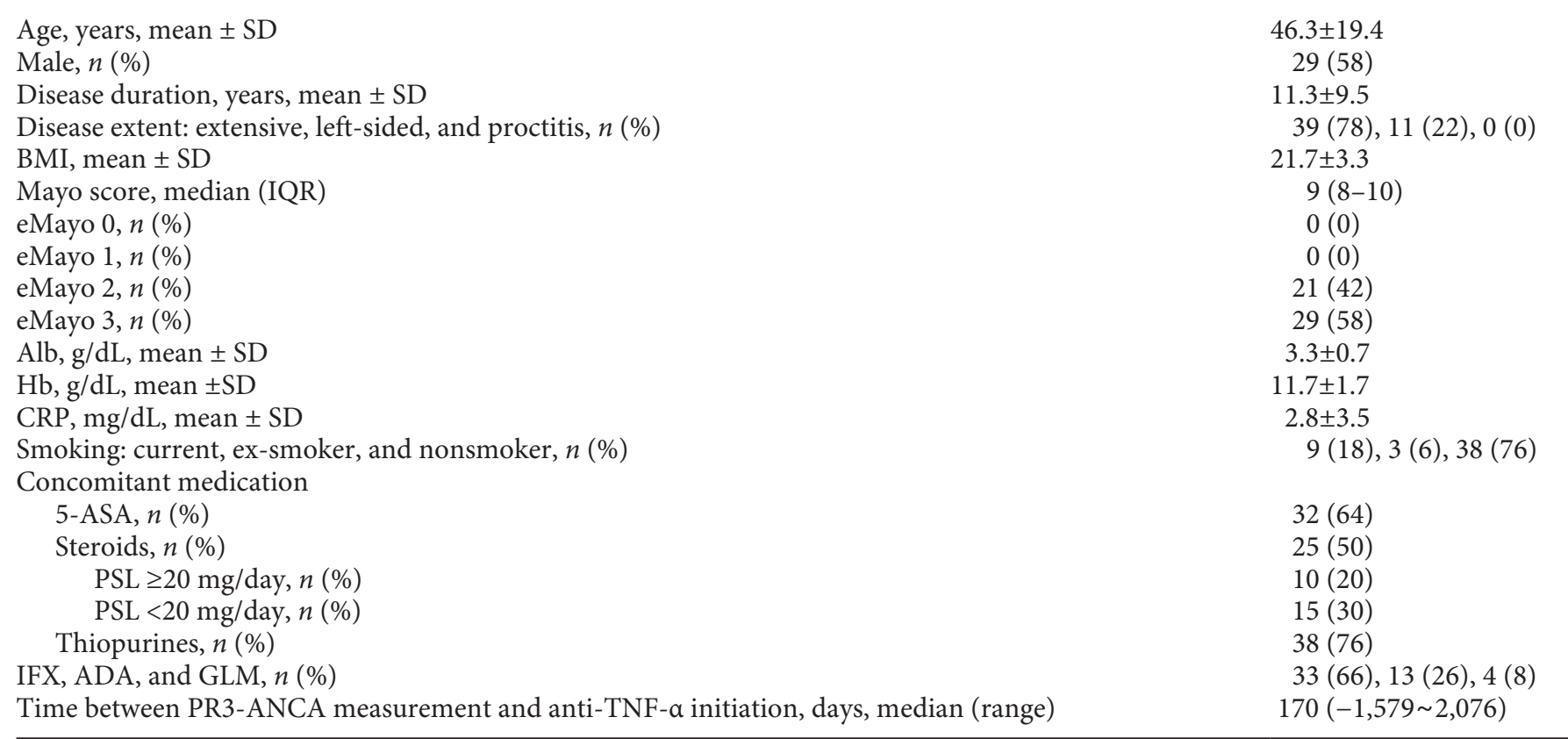

$\mathrm{SD}$, standard deviation; IQR, interquartile range; BMI, body mass index; eMayo, endoscopic Mayo subscore; Alb, albumin; Hb, hemoglobin; CRP, C-reactive protein; 5-ASA, 5-aminosalycilic acid; PSL, predonisolone; IFX, infliximab; ADA, adalimumab; GLM, golimumab; ANCA, anti-neutrophil cytoplasmic antibody.

sis at UC onset. One patient was excluded from analysis because of intolerance to anti-TNF- $\alpha$ agents.

Baseline parameters of the 50 patients are shown in Table 1. The mean age was $46.3 \pm 19.4$ years, 29 (58\%) were male, and 39 (78\%) had extensive colitis. Most patients had moderate colitis with a median Mayo score of 9 (IQR: 8-10). This study did not include UC cases with vasculitis-related extraintestinal symptoms.

\section{Predictors of PNR}

Seventeen $(34 \%)$ of the 50 patients demonstrated PNR at week 6 . Univariate analysis revealed significant differences between patients with and without PNR in $\mathrm{Hb}$ (mean [SD]: 10.9 [1.8] vs. 12.0 [0.7], $p=0.039$ ), smoking cessation history (36\% $[n=6]$ vs. $9 \%[n=3], p=0.044)$, and PR3ANCA positivity $(65 \%[n=11]$ vs. $9 \%[n=3], p<0.001)$ (Table 2). Other factors, including age, sex, disease duration, Mayo score, or CRP, were not significantly different between patients with and without PNR (Table 2). Multivariate analysis using logistic regression analysis showed PR3-ANCA positivity was significantly associated with PNR (odds ratio 19.29, 95\% confidence interval [CI]: 3.30-
$172.67 ; p=0.002)$. No other factors, including BMI, Mayo score, $\mathrm{Hb}$ levels, or smoking cessation history, were significantly associated with PNR (Table 3).

\section{Rates of PNR}

Eleven (78.6\%) of the 14 patients with PR3-ANCA positivity demonstrated PNR, while $6(16.7 \%)$ of the 36 patients with PR3-ANCA negativity demonstrated PNR ( $p=0.0001$, using the $\chi^{2}$ test).

\section{Performance of PR3-ANCA for Predicting PNR}

We examined the performance of PR3-ANCA for predicting PNR: the sensitivity was $64.7 \%$, specificity was $90.9 \%$, positive predictive value was $78.6 \%$, and negative predictive value was $83.3 \%$. The area under the curve of receiver operating characteristics (ROC) analysis was 0.82 (95\% CI: 0.69-0.96). PNR diagnostic performance with best accuracy (threshold $2.45 \mathrm{U} / \mathrm{mL}$ ) obtained by using Youden's method showed that the sensitivity was $82.4 \%$, specificity was $84.8 \%$, positive predictive value was $74.0 \%$, and negative predictive value was $90.3 \%$, respectively. 
Table 2. Characteristics of patients with primary nonresponse and those without

\begin{tabular}{|c|c|c|c|}
\hline Age, years, mean \pm SD & $45.2 \pm 21.5$ & $46.8 \pm 18.6$ & $0.801^{*}$ \\
\hline Male, $n(\%)$ & $10(59)$ & $19(58)$ & $0.933^{* *}$ \\
\hline Disease duration, years, median (IQR) & $10(3-14)$ & $8(6-17)$ & $0.831^{* * *}$ \\
\hline Disease extent: extensive, left-sided, and proctitis, $n(\%)$ & 13 (76), $4(24), 0(0)$ & 26 (79), $7(21), 0(0)$ & $0.851^{* *}$ \\
\hline BMI, median (IQR) & $19.6(18.9-23.1)$ & $21.9(20.0-23.2)$ & $0.073^{* * *}$ \\
\hline eMayo $0, n(\%)$ & $0(0)$ & $0(0)$ & \\
\hline eMayo $1, n(\%)$ & $0(0)$ & $0(0)$ & \\
\hline eMayo $2, n(\%)$ & $5(29)$ & $16(48)$ & $0.196^{* *}$ \\
\hline eMayo $3, n(\%)$ & $12(71)$ & $17(52)$ & $0.364^{* *}$ \\
\hline Alb, g/dL, mean $\pm S D$ & $3.2 \pm 0.7$ & $3.3 \pm 0.7$ & $0.843^{*}$ \\
\hline $\mathrm{Hb}, \mathrm{g} / \mathrm{dL}$, mean $\pm \mathrm{SD}$ & $10.9 \pm 1.8$ & $12.0 \pm 0.7$ & $0.039^{*}$ \\
\hline \multicolumn{4}{|l|}{ Concomitant medication } \\
\hline Steroids, $n(\%)$ & $8(47)$ & $17(52)$ & $0.765^{* *}$ \\
\hline PSL $\geq 20 \mathrm{mg} /$ day, $n(\%)$ & $3(18)$ & $7(21)$ & $0.765^{* *}$ \\
\hline PSL $<20$ mg/day, $n(\%)$ & $5(29)$ & $10(30)$ & $0.948^{* *}$ \\
\hline Thiopurines, $n(\%)$ & $12(71)$ & $26(79)$ & $0.520^{* *}$ \\
\hline IFX, ADA, and GLM, $n(\%)$ & 8 (47), $6(35), 3(18)$ & 25 (78), $7(21), 1(1)$ & $0.072^{* *}$ \\
\hline Time between PR3-ANCA measurement and anti-TNF- $\alpha$ initiation, days, median (range) & $226(-1,579 \sim 2,076)$ & $115(-566 \sim 375)$ & $0.413^{* * *}$ \\
\hline PR3-ANCA,$+ n(\%)$ & $11(65)$ & $3(9)$ & $0.000^{\star \star}$ \\
\hline
\end{tabular}

$\mathrm{SD}$, standard deviation; IQR, interquartile range; BMI, body mass index; eMayo, endoscopic Mayo subscore; Alb, albumin; Hb, hemoglobin; CRP, C-reactive protein; 5-ASA, 5-aminosalycilic acid; PSL, predonisolone; IFX, infliximab; ADA, adalimumab; GLM, golimumab; ANCA, anti-neutrophil cytoplasmic antibody. Bold indicates statistical significance. ${ }^{*} t$ test. ${ }^{* *} \chi^{2}$ test. ${ }^{* * *}$ Wilcoxon rank-sum test.

Table 3. The association between primary nonresponse to anti-TNF- $\alpha$ agents and explanatory variables

\begin{tabular}{lccc}
\hline & Odds ratio & $95 \%$ confidence interval & $p$ value \\
\hline Sex (female) & 0.53 & $0.06-3.60$ & 0.535 \\
BMI & 0.86 & $0.62-1.13$ & 0.321 \\
Mayo score at baseline & 0.93 & $0.44-1.86$ & 0.847 \\
Hemoglobin & 0.92 & $0.53-1.54$ & 0.756 \\
Disease duration & 1.02 & $0.93-1.10$ & 0.650 \\
Smoking cessation & 4.88 & $0.57-51.02$ & 0.154 \\
PR3-ANCA & 19.29 & $3.30-172.67$ & $\mathbf{0 . 0 0 2}$
\end{tabular}

BMI, body mass index; ANCA, anti-neutrophil cytoplasmic antibody. Bold indicates statistical significance.

\section{Discussion}

Herein, we demonstrated PR3-ANCA as a predictor of PNR to anti-TNF- $\alpha$ agents in UC patients. Several studies showed that p-ANCA can predict early clinical response to anti-TNF- $\alpha$ agents in UC patients [14-19]. A metaanalysis demonstrated that p-ANCA-negative patients had an almost two-fold higher response rate to antiTNF- $\alpha$ agents than those positive for $\mathrm{p}$-ANCA. The positive predictive value of $\mathrm{p}$-ANCA for PNR was $41.1 \%$ when p-ANCA was positive, and the positive predictive value of p-ANCA for primary response was $74.0 \%$ when p-ANCA was negative [15]. However, p-ANCA is measured using IIF, sometimes making judgments about positivity and 
negativity difficult. Therefore, judgment often differs depending on measurement facilities. In contrast, PR3ANCA has an advantage over p-ANCA regarding measurement methods. PR3-ANCA is measured using ELISA and CLEIA, which are more sensitive and quantitative than IIF, used to measure p-ANCA $[10,12,13]$. In the present study, we showed the positive predictive value of PR3-ANCA for PNR was 78.6\%, while the negative predictive value was $83.3 \%$. The predictive capacity of PR3ANCA appears sufficient for clinical use. Overall, in clinical practice, PR3-ANCA should be a more useful predictor of PNR to anti-TNF- $\alpha$ in UC patients than p-ANCA.

In the present study, univariate analysis revealed that lower $\mathrm{Hb}$ levels and smoking cessation were associated with PNR. A multicenter retrospective study showed that low $\mathrm{Hb}$ levels correlated with PNR to anti-TNF- $\alpha$ agents in UC patients [23]. Several studies reported that smoking habit is associated with PNR [24]. Furthermore, it was reported that nonsmoking increases the rates of c-ANCA positivity in patients with vasculitis [25]. Given these observations, low $\mathrm{Hb}$ levels and smoking cessation may have confounded with PR3-ANCA in this study. However, multivariate analysis demonstrated PR3-ANCA as a predictor of PNR with high odds ratio, suggesting PR3ANCA is an independent predictor of PNR.

There are reports of effectiveness for anti-TNF- $\alpha$ agents in other ANCA-positive diseases. In AAV, antiTNF- $\alpha$ agents, in addition to standard care, did not affect induction rate, adverse events, activity score, relapse rate, and biomarker levels [26]. In this previous report, antiTNF- $\alpha$ agents had no effect on AAV. In ANCA-positive patients, the mechanism of nonresponse to anti-TNF- $\alpha$ agents is unknown.

This study had several limitations. First, only 14 patients were PR3-ANCA positive, and this small sample size may have been insufficient for multivariate analysis. Second, because of the retrospective nature of this study, we could not exclude the effects of other possible confounding factors on PNR to anti-TNF- $\alpha$ agents. Third, this was a single-center study and the external validity of our findings is unknown. Fourth, the timing of PR3-ANCA measurement varied among subjects. Finally, our study population consisted of patients in whom PR3-ANCA was measured primarily for differential diagnosis of UC. In total, $28 \%$ of patients were PR3-ANCA positive, which was consistent with previously reported positivity rates in UC patients [27]. While this observation suggests our study population was representative of the general UC population, it is unclear whether our findings can be applied to this population. To overcome these limitations, our findings must be validated in prospective large multicenter studies. Despite these limitations, this is the first study to demonstrate that PR3-ANCA can serve as a predictive factor for PNR to anti-TNF- $\alpha$ agents in UC patients.

\section{Acknowledgement}

The authors thank Richard Robins, PhD, from Edanz Group (https://en-author-services.edanzgroup.com/ac) for editing a draft of this manuscript.

\section{Statement of Ethics}

We conducted this study in compliance with the latest version of the Declaration of Helsinki. The study was approved by the institutional ethics committee (the ethics committee of Ofuna Chuo Hospital, No. 2018-011). Patient consent was waived because of the retrospective and observational nature of the study.

\section{Conflict of Interest Statement}

A.Y. received personal fees from Mitsubishi Tanabe Pharma, Janssen Pharmaceutical K.K., and AbbVie Inc. K.M. received personal fees from Mitsubishi Tanabe Pharma, Janssen Pharmaceutical K.K., AbbVie Inc., EA Pharma Co., Ltd., Pfizer Inc., Mochida Pharmaceutical Co.,1k., Alfresa Pharma Co., and Thermo Fisher Scientific K.K.; and research grants from Mitsubishi Tanabe Pharma, Janssen Pharmaceutical K.K., AbbVie Inc., EA Pharma Co., Ltd., Mochida Pharmaceutical Co., Ltd., and Nippon Kayaku Co., Ltd. F.U. received personal fees from Mitsubishi Tanabe Pharma, Janssen Pharmaceutical K.K., AbbVie Inc., and EA Pharma Co., Ltd. Y.E. received personal fees from Mitsubishi Tanabe Pharma, Janssen Pharmaceutical K.K., AbbVie Inc., and EA Pharma Co., Ltd. T.H. received personal fees from Aspen Japan K.K., JIMRO, Mitsubishi Tanabe Pharma, Janssen Pharmaceutical K.K., and Takeda Pharma Co., Ltd.; and research grants from Zeria Pharmaceutical Co., Ltd. T.M. has no conflicts of interest to declare. However, none of the above is relevant to this article.

\section{Funding Sources}

There was no funding for this study.

\section{Author Contributions}

A. Yoshida, K. Matsuoka, and F. Ueno designed the study. Data were collected by A. Yoshida, T. Morizane, and Y. Endo. T. Morizane analyzed the data. A. Yoshida and K. Matsuoka drafted the manuscript and interpreted the data. T. Hibi and K. Matsuoka made critical revisions to the manuscript. All authors reviewed and approved the final version of the manuscript. 


\section{References}

1 Ungaro R, Mehandru S, Allen PB, Peyrin-Biroulet L, Colombel JF. Ulcerative colitis. Lancet. 2017;389(10080):1756-70.

2 Paul R, Sandborn WJ, Feagan BG, Reinisch W, Olson A, Johanns J, et al. Infliximab for induction and maintenance therapy for ulcerative colitis. N Engl J Med. 2005;353:2462-76.

3 Lawson MM, Thomas AG, Akobeng AK. Tumour necrosis factor alpha blocking agents for induction of remission in ulcerative colitis. Cochrane Database Syst Rev. 2006 Jul 19(3):CD005112.

4 Lv R, Qiao W, Wu Z, Wang Y, Dai S, Liu Q, et al. Tumor necrosis factor alpha blocking agents as treatment for ulcerative colitis intolerant or refractory to conventional medical therapy: a meta-analysis. PloS One. 2014;9(1): e86692.

5 Huang X, Lv B, Jin HF, Zhang S. A meta-analysis of the therapeutic effects of tumor necrosis factor- $\alpha$ blockers on ulcerative colitis. Eur J Clin Pharmacol. 2011;67(8):759-66.

6 Singh S, George J, Boland BS, Vande Casteele N, Sandborn WJ. Primary non-response to tumor necrosis factor antagonists is associated with inferior response to second-line biologics in patients with inflammatory bowel diseases: a systematic review and meta-analysis. J Crohns Colitis. 2018;12(6):635-43.

7 Davies DJ, Moran JE, Niall JF, Ryan GB. Segmental necrotising glomerulonephritis with antineutrophil antibody. Possible arbovirus aetiology?. Br Med J. 1982;285:606.

8 van der Woude FJ, Rasmussen N, Lobatto S, Wiik A, Permin H, van Es LA, et al. Autoantibodies against neutrophils and monocytes: tool for diagnosis and marker of disease activity in Wegener's granulomatosis. Lancet. 1985;1(8426):425-9.

9 Falk RJ, Jennette JC. Anti-neutrophil cytoplasmic autoantibodies with specificity for myeloperoxidase in patients with systemic vasculitis and idiopathic necrotizing and crescentic glomerulonephritis. N Engl J Med. 1988;318(25):1651-7.
10 Kallenberg CG, Mulder AH, Tervaert JW. Antineutrophil cytoplasmic antibodies: a still-growing class of autoantibodies in inflammatory disorders. Am J Med. 1992;93(6): 675-82.

11 Gil B, Ben-Zvi I, Furie N, Grossman C. Clinical significance of positive anti-neutrophil cytoplasmic antibodies without evidence of anti-neutrophil cytoplasmic antibodies-associated vasculitis. Int J Rheum Dis. 2019;22: 940-5.

12 Reese GE, Constantinides VA, Simillis C, Darzi AW, Orchard TR, Fazio VW, et al. Diagnostic precision of anti-Saccharomyces cerevisiae antibodies and perinuclear antineutrophil cytoplasmic antibodies in inflammatory Bowel Disease. Am J Gastroenterol. 2006;101:2410-2422.

13 Teresa Arias-Loste M, Bonilla G, Moraleja I, Mahler M, Mieses MA, Castro B, et al. Presence of anti-proteinase 3 antineutrophil cytoplasmic antibodies (anti-PR3 ANCA) as serologic markers in inflammatory bowel disease. Clin Rev Allergy Immunol. 2013;45:109-16.

14 Marc F, Séverine V, Konstantinos HK, Maja N, Gert Van A, Fabian S, et al. Predictors of early response to infliximab in patients with ulcerative colitis. Inflamm Bowel Dis. 2007; 13:123-8.

15 Douglas LN, Nguyen ET, Bechtold ML. pANCA positivity predicts lower clinical response to infliximab therapy among patients with IBD. South Med J. 2015;108:139-43.

16 Leung E. Commentary on "pANCA positivity predicts lower clinical response to infliximab therapy among patients with IBD". South Med J. 2015;108(3):144.

17 Arias MT, Vande Casteele N, Vermeire S, de Buck van Overstraeten A, Billiet T, Baert F, et al. A panel to predict long-term outcome of infliximab therapy for patients with ulcerative colitis. Clin Gastroenterol Hepatol. 2015;13: 531-8.

18 Jürgens M, Laubender RP, Hartl F, Weidinger M, Seiderer J, Wagner J, et al. Disease activity, ANCA, and IL23R genotype status determine early response to infliximab in patients with ulcerative colitis. Am J Gastroenterol. 2010; 105(8):1811-9.
19 Kevans D, Waterman M, Milgrom R, Xu W, Van Assche G, Silverberg M. Serological markers associated with disease behavior and response to anti-tumor necrosis factor therapy in ulcerative colitis. J Gastroenterol Hepatol. 2015;30(1):64-70.

20 Horn MP, Peter AM, Righini Grunder F, Leichtle AB, Spalinger J, Schibli S, et al. PR3ANCA and panel diagnostics in pediatric inflammatory bowel disease to distinguish ulcerative colitis from Crohn's disease. PLoS One. 2018;13:e0208974.

21 Matsuoka K, Kobayashi T, Ueno F, Matsui T, Hirai F, Inoue N, et al. Evidence-based clinical practice guidelines for inflammatory bowel disease. J Gastroenterol. 2018;53(3):305-53.

22 Schroeder KW, Tremaine WJ, Ilstrup DM. Coated oral 5-aminosalicylic acid therapy for mildly to moderately active ulcerative colitis. A randomized study. N Engl J Med. 1987; 317(26):1625-9.

23 Oussalah A, Evesque L, Laharie D, Roblin X, Boschetti G, Nancey S, et al. A multicenter experience with infliximab for ulcerative colitis: outcomes and predictors of response, optimization, colectomy, and hospitalization. Am J Gastroenterol. 2010;105(12):2617-25.

24 Haubitz M, Woywodt A, de Groot K, Haller $\mathrm{H}$, Goebel U. Smoking habits in patients diagnosed with ANCA associated small vessel vasculitis. Ann Rheum Dis. 2005;64(10):1500-2.

25 Mattery DL, Brownfield A, Dawes PT. Relationship between pack-year history of smoking and response to tumor necrosis factor antagonists in patients with rheumatoid arthritis. J Rheumatol. 2009;36:1180-7.

26 Morgan MD, Drayson MT, Savage CO, Harper L. Addition of infliximab to standard therapy for ANCA-associated vasculitis. Nephron Clin Pract. 2011;117(2):c89-97.

27 Takedatsu H, Mitsuyama K, Fukunaga S, Yoshioka S, Yamauchi R, Mori A, et al. Diagnostic and clinical role of serum proteinase 3 antineutrophil cytoplasmic antibodies in inflammatory bowel disease. J Gastroenterol Hepatol. 2018;33(9):1603-7. 